\title{
IMPACTO DA FEBRE AFTOSA NO PREÇO DA ARROBA DO BOI GORDO, RECEBIDO PELO PRODUTOR NO BRASIL ${ }^{1}$
}

\author{
Gibran da Silva Teixeira ${ }^{2}$ \\ Sinézio Fernandes Maia ${ }^{3}$
}

\begin{abstract}
Resumo - Este estudo objetivou identificar alguns fatos que influenciaram os preços da arroba do boi gordo recebido pelo produtor, bem como realizar estimativas desses preços para um período de quatro meses, a partir de maio de 2007. Utilizou-se a metodologia idealizada por Box-Jenkins (1976). Os resultados demonstraram que a série em questão (01/1996-05/2007) apresentou uma quebra estrutural no período referente a janeiro de 2004, fato que levou ao corte desta, sendo considerado na análise o intervalo de fevereiro de 2004 a maio de 2007, apresentando 40 observações. Alguns acontecimentos podem ter influenciado os preços da arroba do boi gordo naquele momento; destes, um que merece destaque é o fato de que, no período foram detectados dois focos de febre aftosa nos estados do Amazonas e do Pará, o que acarretou o embargo das exportações para Rússia.
\end{abstract}

Palavras-Chave: Preço, boi gordo, febre aftosa.

\section{Introdução}

No Brasil, os primeiros focos de febre aftosa foram constatados em 1895 e, até a década de 80, foi considerada uma doença endêmica. A partir de 1970, a preocupação com a qualidade das vacinas utilizadas e o controle das áreas infectadas pelo estudo do trânsito de animais, comparado à ocorrência de focos, permitiram avançar no combate à doença. O período de 1980 a 1988 foi marcado pela redução dos focos,

Recebido em: 26/05/08; Aceito em: 19/08/03.

Mestrando em Economia, PPGE/ UFPB, graduado em Economia pela FURG / 2004.Email: gsteixeira2000@yahoo.com.br

Professor Adjunto, Dept. de Economia e Coordenador do PPGE, UFPB. Doutor em Economia pelo Pimes (UFPE) / 2001. 
por meio de programas para erradicação em nível regional e também por meio da estrutura de produção, fatores considerados determinantes no combate à doença (Lima et al., 2005).

Na medida em que houve a conscientização da importância do controle e a erradicação da doença, o reconhecimento das áreas livres de aftosa pelo Escritório Internacional de Epizootias (OIE) foi um passo importante, conseguido pelo Brasil, para promover a exportação de carne bovina. A partir de 1999, as exportações passaram a apresentar tendência fortemente ascendente, e, mesmo sabendo que a taxa de câmbio é responsável pela maior parte desse resultado, certamente o equacionamento da questão sanitária permitiu atender a um pré-requisito do mercado para viabilizar esse potencial de comércio (Miranda, 2001).

No histórico recente de surtos de febre aftosa no Brasil, os piores anos foram os de 2000 e 2001, quando se identificaram, respectivamente, 47 e 37 casos (Figura 1), sendo que, nesses anos, milhares de cabeça de gado foram exterminadas, causando grandes perdas, tanto econômico quanto social, para o País. No entanto, dois focos de febre aftosa foram encontrados novamente em 2004 e quatro em 2005. Esse reaparecimento da doença levou a Rússia, em 2004, a suspender temporariamente suas importações, o que resultou em novos prejuízos à economia brasileira.

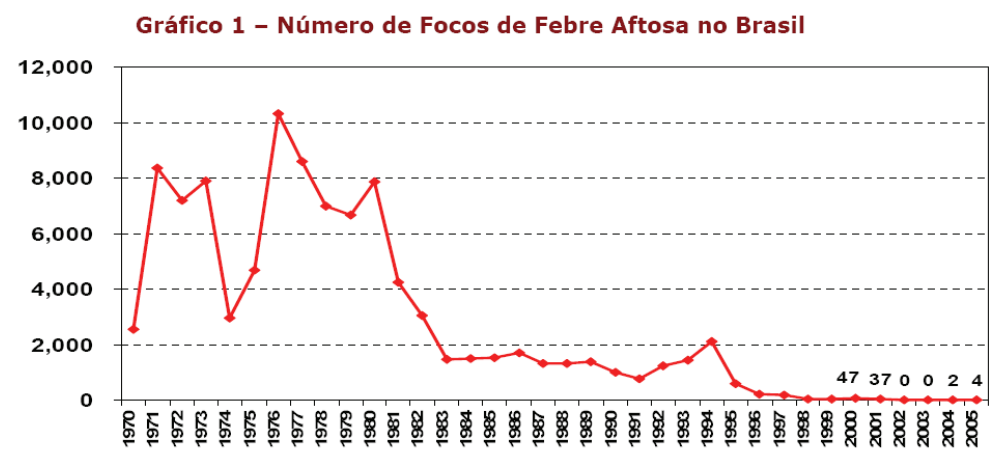

Fonte: Lima et al. (2005).

Figura 1 - Número de focos de febre aftosa no Brasil. 
Dentre os maiores mercados consumidores de carne bovina do mundo, destacam-se os Estados Unidos, que importam cerca de 1.1 milhão de toneladas, em média, anualmente. No entanto, com relação ao destino da maioria das exportações de carne bovina oriunda do Brasil, ressaltase a presença da Rússia, que é o maior importador e foi responsável por $16 \%$ das exportações nacionais em 2004, conforme figura abaixo.

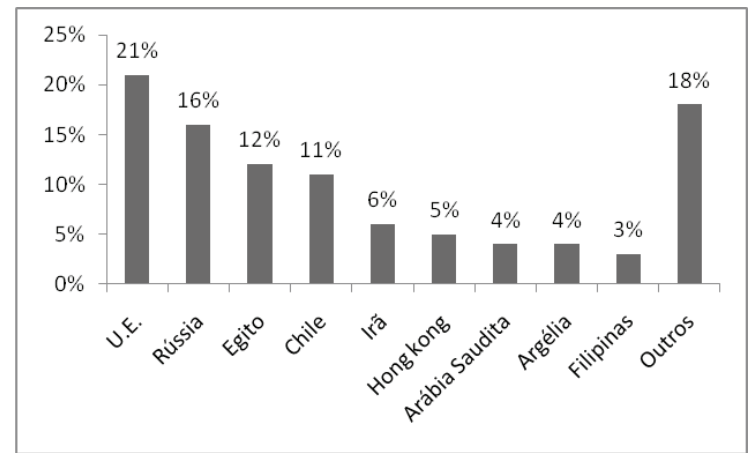

Fonte: Lima et al. (2005).

Figura 2 - Destino das exportações brasileiras de carne bovina in natura, 2004.

Diante do contexto de instabilidade no setor exportador de carne bovina (em relação à saúde animal) e dos possíveis efeitos gerados sobre os preços recebidos pelos produtores, é que surge o questionamento: Até que ponto o cenário externo está influenciando as cotações dos preços da arroba do boi gordo recebido pelo produtor no mercado interno?

Imbuído nessa questão, o estudo objetivou identificar o impacto da febre aftosa no preço da arroba do boi gordo recebido pelo produtor no Brasil, bem como realizar estimativas ex-ante para esses preços, no intervalo de junho de 2007 a setembro de 2007, como forma de corroborar a eficiência do modelo.

Além desta introdução, o artigo está estruturado da seguinte forma. Na seção 2, destaca-se a metodologia Box-Jenkins; Na seção 3, apresenta- 
se o estudo da série dos preços da arroba do boi gordo recebido pelo produtor nacional, abordando alguns pontos relacionados com aparições da febre aftosa e seus possíveis impactos nos preços; por fim, têm-se as considerações finais.

\section{Metodologia}

A metodologia utilizada fundamenta-se na construção de modelos univariados de previsão de preços com base em dados de séries temporais. Os dados utilizados foram os preços da arroba ${ }^{4}$ do boi gordo, oriundos da Fundação Getúlio Vargas (FGV-Agroanalysis), tendo como intervalo de tempo o período de janeiro de 1996 a maio de 2007. A atualização dos preços foi realizada com base no Índice Geral de Preços Disponibilidade Interna (FGV/IGP-DI), visto que esse índice constitui uma média de outros índices, como o de Preços por Atacado (IPA), o Índice Nacional de Custo da Construção (INCC) e o Índice de Preço ao Consumidor (IPC), caracterizado por abranger os diversos setores da economia. A seguir, será tratada a metodologia Box-Jenkins.

\subsection{Modelo Econométrico}

Segundo Morettin e Toloi (2006), antes de verificar se um modelo será auto-regressivo (AR), com integração (I) e com médias móveis (MA), é preciso realizar alguns testes. O modelo de raiz unitária é de fundamental importância para os modelos de séries temporais, pois, a partir dele, é que se definirão quantas diferenças um modelo terá que ser integrado para que a série de dados analisadas se torne estacionária ${ }^{5}$.

\footnotetext{
Normalmente, a cotação referente ao boi gordo tem como unidade de medida a arroba, que, se for transformada em quilogramas, representa $15 \mathrm{~kg}$.

Para Greene (2003), uma série será estacionária quando cada elemento do período analisado apresentar $\varepsilon(\varepsilon)$ $=0, \varepsilon\left(\varepsilon^{2}\right)=o^{2}$ e $\operatorname{Cov}[\varepsilon, \varepsilon]=0$ para todo $\mathrm{s} \neq \mathrm{t}$. Mais a frente será destacado o teste de raiz unitária, definidó por Dickey-Fuller (1976).
} 
Com a intenção de realizar a identificação do melhor modelo ARIMA, torna-se prudente fazer uma análise teórica das formas que esse modelo pode admitir, dependendo se a série utilizada possui ou não raiz unitária. Os Modelos ARIMA são utilizados quando a série possui estacionariedade.

Se as observações $y_{t}$ forem geradas tanto pela média ponderada das $p$ primeiras observações próximas anteriores da variável, quanto pela média ponderada dos $q$ primeiros valores passados de um processo de "ruído branco", e ainda pertencerem a um processo não-estacionário, será necessário diferenciar a série original dos dados $d$ vezes, até se obter uma série estacionária. Assim define-se o modelo ARIMA.

Para Morettin e Toloi (2006), intuitivamente, um processo Z será estacionário se ele se desenvolver no tempo, de modo que a escolha de uma origem dos tempos não é importante. Em outras palavras, as características de $\mathrm{Z}(\mathrm{t}+\tau)$, para todo $\tau$, são as mesmas de $\mathrm{Z}(\mathrm{t})$. Tecnicamente, há duas formas de estacionariedade: fraca (ou de segunda ordem) e estrita (ou forte), definidas a seguir.

(i) Um processo estocástico ${ }^{6} \mathrm{Z}=\{\mathrm{Z}(\mathrm{t}), \mathrm{t} \in \mathrm{t}, \mathrm{T}\}$ será estritamente estacionário se todas as distribuições finito-dimensionais permanecerem as mesmas, sob translações no tempo, ou seja,

$\mathrm{F}\left(\mathrm{z}_{1}, \ldots, \mathrm{z}_{\mathrm{n}} ; \mathrm{t}_{1}+\tau, \ldots, \mathrm{t}_{\mathrm{n}}+\tau\right)=\mathrm{F}\left(\mathrm{z}_{1}, \ldots, \mathrm{z}_{\mathrm{n}} ; \mathrm{t}_{1} \ldots, \mathrm{t}_{\mathrm{n}}\right)$, para quaisquer $\mathrm{t}_{1}, \ldots$, $\mathrm{t}_{\mathrm{n}}, \tau$ de $\mathrm{T}$.

Isto significa, em particular, que todas as distribuições unidimensionais são invariantes sob translações do tempo, logo, a média $\mu(\mathrm{t})$ e a variância $\mathrm{V}(\mathrm{t})$ são constantes, isto é,

$\mu(t)=\mu, V(t)=\sigma^{2}$,

\footnotetext{
Segundo Greene (2003), seja $T$ um conjunto arbitrário. Um processo estocástico é uma família $Z=\{Z(t), t$

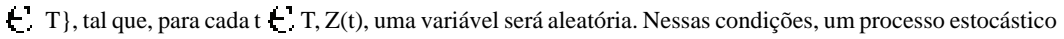
é uma família de variáveis aleatórias, que, supõe-se, sejam definidas num mesmo espaço de probabilidade $(\Omega, Ж, \bar{F})$.
} 
para todo $t \in$ T. Sem perda de generalidade, pode-se supor que $\mu=0$; caso contrário, considere o processo

$\{\mathrm{Z}(\mathrm{t})-\mu\}$

Como o objetivo é caracterizar os processos estocásticos por meio de pequeno número de função de distribuição de probabilidade ou de momentos, restringiu-se aos momentos de primeira e segunda ordens.

(ii) $\mathrm{Um}$ processo estocástico $\mathrm{Z}=\{\mathrm{Z}(\mathrm{t}), \mathrm{t} \in \mathrm{T}\}$ será fracamente estacionário ou estacinário de segunda ordem (ou em sentido amplo) se, e somente se:

(a) $\mathrm{E}\{\mathrm{Z}(\mathrm{t})\}=\mu(\mathrm{t})=\mu$, constante, para todo $\mathrm{t} \in \mathrm{T}$;

(b) $\mathrm{E}\left\{\mathrm{Z}^{2}(\mathrm{t})\right\}<\infty$, para todo $\mathrm{t} \in \mathrm{T}$;

(c) $\gamma\left(\mathrm{t}_{1}, \mathrm{t}_{2}\right)=\operatorname{Cov}\left\{\mathrm{Z}\left(\mathrm{t}_{1}\right), \mathrm{Z}\left(\mathrm{t}_{2}\right)\right\}$ é uma função de $\left[\mathrm{t}_{1}-\mathrm{t}_{2}\right]$.

Se, após extrair-se a primeira diferença, a série apresentar um valor calculado relacionado com a estatística de Dickey-Fuller menor, em valores absolutos, do que o valor crítico a 5\%, a série deverá ser diferenciada novamente, porém algumas variáveis poderão apresentar característica de não-estacionariedade mesmo após realizar-se a segunda diferença, o que impossibilita a utilização de modelos Box Jenkins.

Em suma, o teste de Dickey e Fuller é realizado da seguinte maneira: se a série for estacionária, $(d=0)$; se não-estacionária com uma ou duas diferenças; $d=1$ ou $d=2$. A hipótese básica do teste $\left(\mathrm{H}_{\mathrm{o}}\right)$ é de que a série seja não-estacionária, e a hipótese alternativa $\left(\mathrm{H}_{\mathrm{a}}\right)$ é de que esta seja estacionária. O teste é realizado pela relação estabelecida entre os valores absolutos das estatísticas $\tau_{\text {calculado }}$ e $\tau_{\text {crítico }}$. Se $\tau_{\text {calculado }}<\tau_{\text {críico }}$ (em valores absolutos), aceita-se $\mathrm{H}_{\mathrm{o}}$, e a série será não-estacionária; caso contrário, rejeita-se $\mathrm{H}_{\mathrm{o}}$ e a série será estacionária. 
O número "d", de diferenças necessárias para tornar a série estacionária, é denominado ordem de integração. A inclusão do termo de ordem de integração permite que sejam utilizados os modelos $\operatorname{ARIMA}(\mathrm{p}, \mathrm{d}, \mathrm{q})^{7}$, dados pela equação:

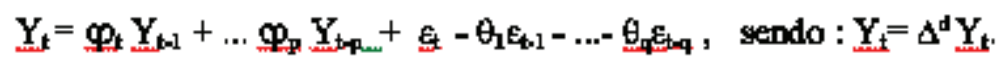

O modelo ARIMA (p,d,q), dado pela equação acima, pode ser reescrito, ao se utilizar o operador de defasagem L:

$$
\left(1-\Phi_{t} L-\ldots-\Phi_{p} L^{P}\right) \underline{Y}_{t}=\left(1-\theta_{1} L-\ldots-\underline{\theta}_{q} L T \underline{\varepsilon_{t}} \quad \text { cont: } \underline{Y}_{t}=(1-L)^{d} \underline{Y}_{t}\right.
$$

$$
\varphi(L)(1-L){ }^{d} Y_{t}=\theta(L) g_{t}
$$

Segundo Costa e Maia (2008), o teste de Zivot e Andrews (1992), referente à presença de raiz unitária e de quebra estrutural, questiona a suposição de exogeneidade da quebra estrutural do teste de Perron (1989) e testa a hipótese nula de raiz unitária contra a hipótese alternativa de estacionariedade com mudança estrutural para algum ponto, no tempo, não conhecido, ou seja, o ponto de quebra estrutural é estimado e não fixado, como no teste de Perron. Dessa forma, a hipótese nula é dada por

$$
y=\mu+y_{t-1}+e_{t}
$$

Nesse teste, deve-se escolher um ponto de quebra estrutural que forneça o menor resultado favorável à hipótese nula (equação 4), utilizando-se a estatística ${ }_{n^{i}}(\lambda)$, isto é, $\lambda$ é escolhido para minimizar a estatística

\footnotetext{
Segundo Morettin e Toloi (2006), esses coeficientes são definidos da seguinte maneira: p é o número de defasagens que a variável irá sofrer ao longo da estimativa; d, número de diferenças necessárias para que a série torne-se estacionária; e q, o número de médias móveis que serão utilizadas na estimativa.
} 
unilateral $t$-Student por testar $\alpha^{j}=1(i=A, B, C)^{8}$, quando os valores pequenos da estatística conduzem à rejeição da hipótese nula. Considerando-se que $\hat{\lambda}_{\text {inf }}^{i}$ represente tal valor de minimização para o

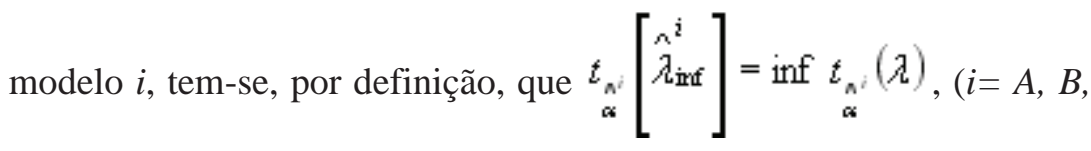
$C$ ), em que $\Lambda$ é um subconjunto específico fechado de [0,1] (Zivot; Andrews, 1992).

A partir da equação (4), Zivot e Andrews usaram as seguintes equações para testar a raiz unitária:

$$
\begin{aligned}
& y_{t}=\hat{n}^{A}+\hat{\theta}^{A} D U(\hat{\lambda})+\hat{\beta}^{A} t+\hat{\alpha}^{A} y_{t-1}+\sum_{j=1}^{k} c_{j} \Delta_{t-j}+\hat{e}_{t} \\
& y_{t}=\hat{\mu}^{B}+\hat{\beta}^{B} t+\hat{\gamma}^{B} D T_{t}^{*}(\hat{\lambda})+\hat{\alpha}^{B} y_{t-1}+\sum_{j=1}^{k} \hat{c}_{j}^{B} \Delta y_{t-j}+\hat{e}_{t} ; \\
& y_{t}=\hat{\mu}^{c}+\hat{\theta}^{c} D U(\hat{\lambda})+\hat{\beta} t+\hat{\gamma}^{c} D T_{i}^{*}(\hat{\lambda})+\hat{\alpha}^{c} y_{t-1}+\sum_{j=1}^{k} \hat{c}_{j}^{c} \Delta y_{t-j}+\hat{e}_{i} ;
\end{aligned}
$$

em que $D U_{t}(\lambda)=1$, se $t>T \lambda ; 0$, caso contrário; $D T^{*}(\lambda)=t-T \lambda$, se $t>T \lambda$; 0 , caso contrário. Colocou-se "chapéu" sobre o parâmetro de $\lambda$ nas equações

\footnotetext{
Nesse caso, as letras A, B, C estão sinalizando os diferentes tipos de quebra estrutural que podem ocorrer, sendo A um modelo com uma mudança no intercepto; B, com mudança na inclinação; e C, com mudança em ambos.
} 
(5), (6) e (7), para enfatizar que eles correspondem aos valores estimados da quebra estrutural. Nesse contexto, a interpretação do teste de raiz unitária, de Perron, é feita da seguinte forma: rejeitar-se-á a hipótese

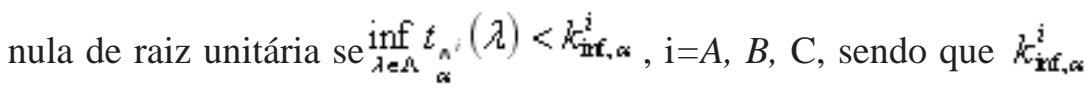
representa o valor crítico tabelado da estatística $\inf _{\lambda \in \mathbb{\Lambda}} t_{\mathrm{n}^{i}}(\lambda)^{9}$.

Na próxima seção serão analisados os dados utilizados no trabalho, bem como o modelo identificado para realizar a previsão do preço da arroba do boi gordo nos meses de 06/2007 a 09/2007.

\section{Análise dos resultados}

Fundamentada na série deflacionada do preço do boi gordo entre 19962007, pretende-se verificar o impacto da febre aftosa nos preços da arroba do boi gordo recebido pelo produtor, além de realizar uma previsão desses preços para os 4 (quatro) meses seguintes, por meio do modelo ARMA (ARIMA), realizando o diagnóstico do(s) modelos(s) estimado(s) e decidindo, portanto, a melhor especificação para a previsão.

Com relação às estatísticas da série, algumas observações podem ser colocadas. O preço médio da arroba do boi gordo (em valor real, com base em maio/2007), durante o período pesquisado, foi de $\mathrm{R} \$ 61,32 \mathrm{e}$ chegou a atingir um valor máximo de $\mathrm{R} \$ 71,19$ (novembro/1999) e um valor mínimo de $\mathrm{R} \$ 50,04$ (julho/2006). O desvio-padrão desse preço foi de $\mathrm{R} \$ 5,62$.

Os valores críticos para os testes de raiz unitária com quebra estrutural podem sem encontrados em Perron (1989) e Zivot e Andrews (1992). 


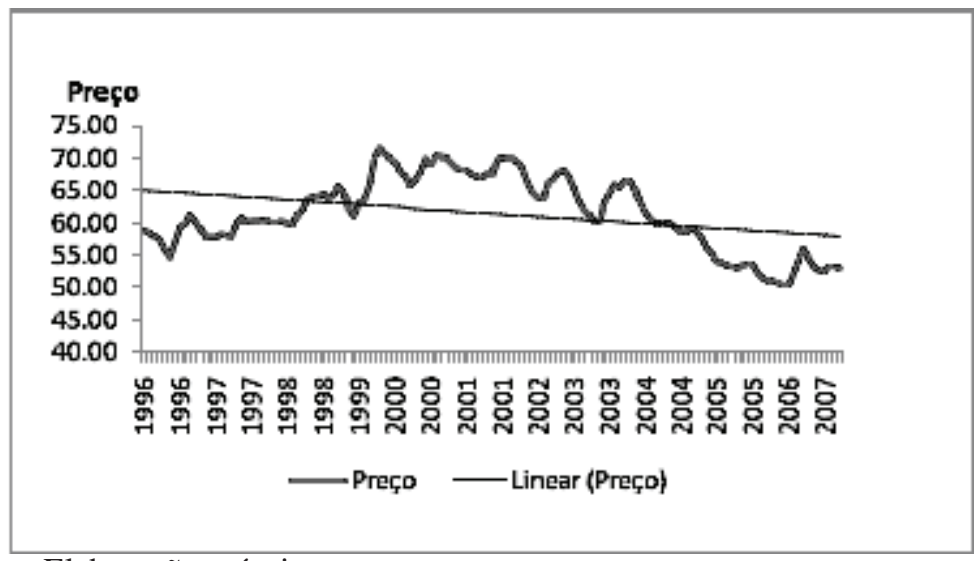

Fonte: Elaboração própria.

Figura 3 - Preços da arroba do boi gordo ao produtor (1996-2007).

Na Figura 3 identifica-se o comportamento dos preços da arroba do boi gordo ( $\mathrm{R} \$ / 15 \mathrm{~kg}$ ) e verifica-se que, ao longo do tempo, este segue um comportamento oscilatório, característica geralmente peculiar a preços de produtos agrícolas, em decorrência da sazonalidade.

Após a identificação do histórico da série, torna-se necessário realizar a decomposição desta, retirando os componentes sazonais, cíclicos, irregular e tendência. Na Figura 4, abaixo, esses componentes são ressaltados. 

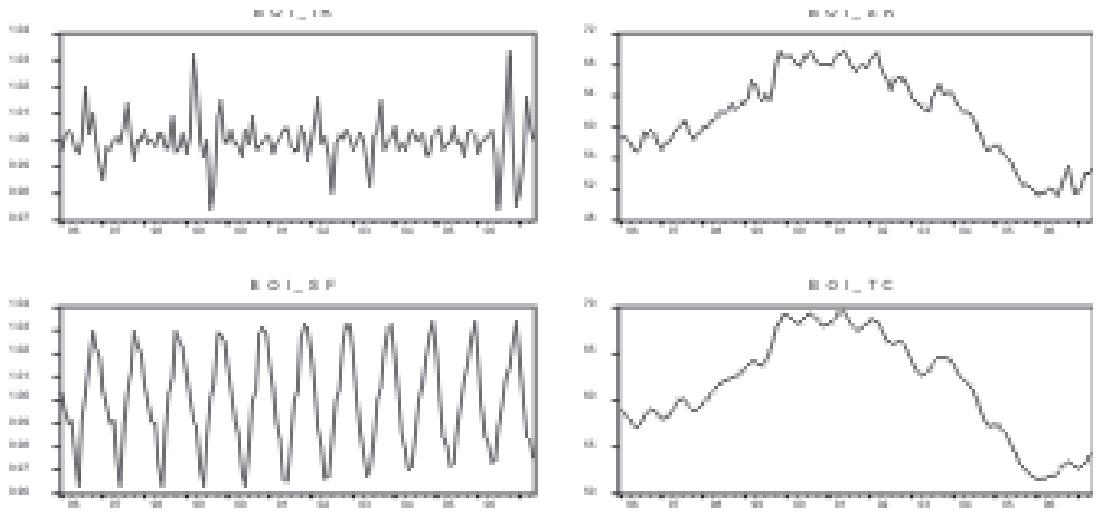

Fonte: Elaboração própria.

Figura 04 - Estudo da série: Preços da arroba do boi gordo ao produtor (1996-2007).

Segundo Lima Neto e Loureiro (2003), o movimento cíclico compreende as oscilações de longo prazo que podem ocorrer em torno de uma linha de tendência. Tais movimentos podem ser, ou não, periódicos e somente são considerados quando ocorrem depois de um intervalo de tempo superior a um ano. Pela análise dos componentes referentes tanto à sazonalidade quanto à tendência, uma quebra nos preços acentua-se no final de 2001.

No entanto, ao comparar os casos de febre aftosa com o comportamento dos preços, verificam-se quebra de tendência crescente nos preços da arroba e certa estabilização iniciada em 2000 até fins de 2001. Esse momento marca o auge dos focos da febre aftosa no País, quando foram identificados, respectivamente, 47 e 37 focos, o que fez com que milhares de cabeças de gado fossem sacrificadas principalmente nos estados do Mato Grosso, Mato Grosso do Sul, Rio Grande do Sul e Santa Catarina, maiores produtores de carne bovina do Brasil. Entretanto, a partir de 2002, a queda nos preços da arroba do boi gordo foi se acentuando até chegar a janeiro de 2004, quando começaram a ter forte queda, o que pode estar associado ao reaparecimento da febre aftosa nos estados do Amazonas e do Pará, fator que levou a Rússia a embargar as importações 
de carne in natura tanto bovina quanto suína. No entanto, para verificar se realmente ocorreu nova quebra estrutural a partir de 2004:01, utilizou-se o teste de Zivot e Andrews (1992).

O teste realizado na quebra estrutural apresentou a estatística $\mathrm{T}=-2.74900$ para 2004:01, e os valores críticos foram, a 1\% de significância, de -4.38, e a 5\% de significância, de -3.60 . Dessa forma, em virtude de a série apresentar quebra estrutural, realizou-se um corte nesta e considerouse, para fins de análise, o intervalo de fevereiro de 2004 a maio de 2007. Com isso, a série passou a ter 40 observações, bem como comportamento decrescente. A figura, a seguir, retrata o estudo da nova série com seus componentes.
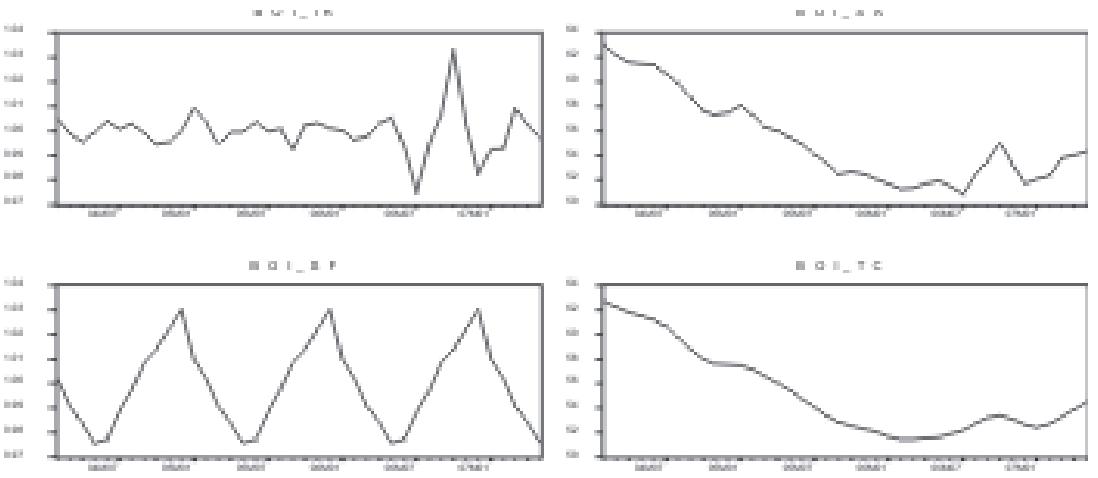

Fonte: Elaboração própria.

Figura 05 - Estudo da segunda série: Preços da arroba do boi gordo ao produtor (2004-2007).

Como o objetivo deste trabalho é tanto identificar a relação entre o número de focos de febre aftosa no País e o preço da arroba do boi gordo, quanto realizar uma previsão para a série de preços da arroba do boi gordo, após o estudo da série, ao retirar os componentes acima citados, bem como subtrair possíveis quebras estruturais, parte-se para a análise da estacionariedade, visto que, para aplicação de qualquer arcabouço 
metodológico definido por Box-Jenkins, é imprescindível que os resíduos sejam estacionários e ruídos brancos.

Para identificar se uma série possui, ou não, raiz unitária, pode-se testála de duas maneiras diferentes; ou por meio dos correlogramas, via funções de autocorrelação e autocorreção parcial, ou por meio do método da estatística de Dickey-Fuller " $\tau$ ". Primeiramente, será analisado o correlograma das funções de autocorrelação e autocorrelação parcial em nível e, logo após, em primeira diferença.
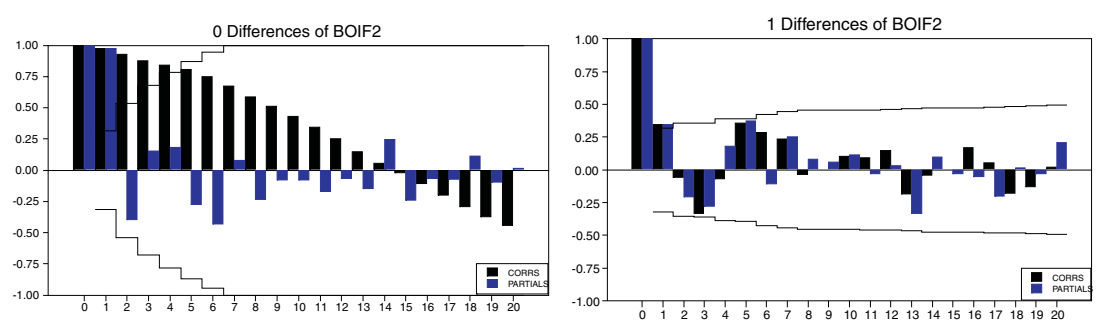

Fonte: Elaboração própria.

Figura 06 - Correlograma da segunda série: Preços da arroba do boi gordo ao produtor (2004-2007).

Pela análise das Figuras $6 a$ e 6b, percebe-se que a série é fracamente estacionária, e, pela análise da função de autocorrelação parcial, visualizase que o modelo estrutural sugerido é um ARIMA $(2,1,2)$, ou seja, um modelo autoregressivo de ordem dois ou $\mathrm{AR}=2$, com duas médias móveis ou $M A=2$. Visto que a série é estacionária em primeira diferença, temse uma integração ou $\mathrm{I}=1$; no entanto, antes de começar a destacar os modelos, é importante constatar, pelo teste de Dickey-Fuller Ampliado, se a série realmente é estacionária em primeira diferença. 
Tabela I - Testes Dickey-Fuller Ampliado (ADF)

\begin{tabular}{|c|c|c|}
\hline ESTATÍSTICA ( $\tau)$ & $\begin{array}{l}\text { SÉRIE EM } \\
\text { NIVEL }\end{array}$ & $\begin{array}{c}\text { SÉRIE EM PRIMEIRA } \\
\text { DIFERENÇA }\end{array}$ \\
\hline$\tau$ CALCULADO & & \\
\hline$\overline{\tau \text { CRÍTICO } 1 \%}$ & & -4.38 \\
\hline$\tau$ CRÍTICO $5 \%$ & & -3.60 \\
\hline$\tau$ CRÍTICO 10\% & & -3.24 \\
\hline \multicolumn{3}{|c|}{ Fonte: Elaboração própria. } \\
\hline \multicolumn{3}{|c|}{$\begin{array}{l}\text { De acordo com os valores observados na tabela anterior, pode-se constatar } \\
\text { que a série é raiz unitária em nível e estacionária em primeira diferença, } \\
\text { visto que o valor calculado da série em nível é maior do que qualquer } \\
\text { valor crítico de ô, e, em primeira diferença, o valor calculado é menor } \\
\text { que qualquer valor crítico tabelado. A partir de então, definem-se os } \\
\text { modelos que poderão ser utilizados na previsão dos preços. }\end{array}$} \\
\hline \multicolumn{3}{|c|}{$\begin{array}{l}\text { A partir dos modelos selecionados, identificou-se que o ARIMA }(2,1,2) \\
\text { foi o que obteve os menores valores das estatísticas AIC e SBC. Em } \\
\text { virtude disso, esse modelo foi escolhido para estimar os preços da arroba } \\
\text { do boi gordo recebido pelos produtores no Brasil. Porém, é preciso } \\
\text { destacar que, pela análise do erro quadrado médio da regressão, o } \\
\text { melhor modelo seria o ARIMA }(0,1,2) \text {, ou seja, um modelo com duas } \\
\text { médias móveis e com uma diferenciação. Dada a ocorrência desse } \\
\text { fato, parte-se para análise dos coeficientes dos modelos candidatos. }\end{array}$} \\
\hline
\end{tabular}


TABELA II - Análise do modelo ARIMA $(2,1,2)$

\begin{tabular}{lcccc}
\hline Variável & Coeficiente & Erro padrão & T-Stat & Prob \\
& & & & \\
\hline AR (1) & 0.144235872 & 0.082382078 & 1.75082 & 0.08926994 \\
AR (2) & -0.277113542 & 0.064533762 & -4.29409 & 0.00014497 \\
MA (1) & 0.727748209 & 0.201267618 & 3.61582 & 0.00098655 \\
MA (2) & 1.744837286 & 0.258262118 & 6.75607 & 0.00000011 \\
\hline
\end{tabular}

Fonte: Elaboração própria.

Segundo os valores mencionados, nota-se que quase todos os coeficientes foram significativos a $5 \%$. No entanto, o coeficiente relacionado com o $\operatorname{AR}(1)$ foi o único que apresentou probabilidade maior do que 5\% e não se mostrou significativo a esse percentual; no entanto, se for considerando um grau de significância de $10 \%$, todos os coeficientes foram significativos. Análisados os coeficientes do primeiro modelo, parte-se para análise do segundo, destacado na tabela a seguir.

TABELA III - Análise do modelo ARIMA $(0,1,2)$

\begin{tabular}{lrrrc}
\hline Variável & Coeficiente & Erro padrão & T-Stat & Prob \\
& & & & \\
MA (1) & 0.5784328466 & 0.1426602838 & 4.05462 & 0.00024819 \\
MA(2) & 0.5109119934 & 0.1433172231 & 3.56490 & 0.00102515 \\
\hline
\end{tabular}

Fonte: Elaboração própria.

Pelos resultados dos coeficientes encontrados na tabela anterior, constatase que os dois coeficientes, tanto referente ao MA(1) quanto ao MA(2), foram significativos a $5 \%$ e $10 \%$, fator que reforça a necessidade da comparação dos resultados da previsão entre estes, realizada na tabela seguinte. 
TABELA IV - Resultados ARIMA $(2,1,2)$ e $(0,1,2)$ Preços ex-post

\begin{tabular}{cccc}
\hline Período & $\begin{array}{c}\text { Preço estimado } \\
\text { ARIMA (2,1,2) }\end{array}$ & $\begin{array}{c}\text { Preço real } \\
\text { (R\$/ } / \mathbf{l n g})\end{array}$ & $\begin{array}{c}\text { Preço estimado } \\
\text { ARIMA (0,1,2) }\end{array}$ \\
$2006: 12$ & 52.0243676947 & 51.74882 & 52.2350343824 \\
$2007: 01$ & 51.9290875892 & 52.06474 & 51.1185319191 \\
$2007: 02$ & 52.2596950193 & 52.36832 & 51.1185319191 \\
$2007: 03$ & 52.3337838780 & 53.79939 & 51.1185319191 \\
$2007: 04$ & 52.2528543532 & 54.01525 & 51.1185319191 \\
$2007: 05$ & 52.2206503866 & 54.35483 & 51.1185319191 \\
\hline
\end{tabular}

Fonte: Elaboração própria.

A previsão ex-post é realizada para gerar valores dentro do próprio período. A idéia é que, quanto melhores forem essas previsões, melhor será o modelo estimado. Portanto, o EQM da previsão (que é igual à média do quadrado da diferença entre o valor real observado na amostra e cada valor previsto ex-post) é uma medida formal da qualidade das previsões ex-post. Assim, para efeitos de comparabilidade, quanto menor o EQM, melhor será o grau de ajustamento do modelo aos dados da série temporal. Porém, como no presente estudo as estatísticas AIC e SBC foram as menores em um único modelo, não foi necessário analisar o erro quadrado médio dos outros modelos como critério de escolha. No entanto, como tentativa de identificar a melhor previsão, colocou-se um segundo modelo com menor EQM, para critério de comparação.

Assim, ao identificar os resultados, verificou-se que o modelo ARIMA $(2,1,2)$ apresentou melhor previsão ex-post que o ARIMA $(0,1,2)$, razão por que foi escolhido para a realização da estimativa ex-ante no primeiro modelo, como apresentado na tabela seguinte. 
TABELA V - Resultados da Previsão ex-ante

Período

2007:06

2007:07

2007:08

2007:09
Preço estimado ARIMA $(2,1,2)$

52.2384320866

52.2499210009

52.2466505646

52.2429951166

Fonte: Elaboração própria.

Os valores da tabela acima mostram que o modelo usado nas estimativas dos preços captou tendência de baixa nestes, mas com certa estabilização nos meses previstos. A seguir, serão discutidos os principais resultados encontrados ao longo do trabalho, destacando-se a questão dos embargos comerciais em razão do aparecimento dos focos de febre aftosa e dos possíveis impactos que isso pode ter gerado no preço da arroba do boi gordo recebido pelo produtor no Brasil.

\section{Considerações Finais}

Ao analisar a série dos preços da arroba do boi gordo recebido pelo produtor nacional, identificou-se a existência de quebra estrutural em 01/ 2004, constatação que foi possível por meio do teste de quebra estrutural endógena, de Zivot e Andrews (1992). Com base nessa quebra, tem-se que o preço da arroba do boi gordo ao produtor pode ter sido influenciado pelo embargo das importações da Rússia, em razão do surgimento de dois focos de febre aftosa no Amazonas e no Pará, nesse período. No entanto, é importante destacar que, no período de 2000 e 2001, quando ocorreram os maiores focos de febre aftosa no Brasil, nos últimos tempos, os preços da arroba do boi gordo recebido pelo produtor no país mantiveram-se estabilizados, freando a tendência de alta anteriormente analisada.

Essa constatação credencia o estudo a conjecturar que, quando ocorreu o maior surto de febre aftosa da histórica recente do país, ocasião em 
que milhares de cabeça de gado foram sacrificadas, o que reduziu a oferta nacional, a demanda interna manteve o preço recebido pelo produtor, já que a demanda externa foi altamente comprometida pelos embargos gerados por países importadores como Rússia e Reino Unido.

Entretanto, já em 2004, quando houve o reaparecimento da febre nos estados do Amazonas e Pará, que não são estados com expressão na produção de carne bovina no País, mas mesmo assim promoveram um novo embargo da Rússia e também criaram incerteza no mercado internacional acerca da possibilidade de um novo surto de aftosa no Brasil, a oferta interna manteve- se constante. Esse fato foi decorrente do quase insignificante número de abate de animais, ou seja, com a queda da demanda internacional e com a demanda interna constante, o volume ofertado foi maior que o normal, o que pode ter gerado excesso de oferta e, com isso, queda brusca nos preços, que se seguiu no restante do período analisado.

Com relação aos preços estimados por meio do modelo ARIMA $(2,1,2)$, considerado como o de melhor ajuste, verifica-se que este captou tendência de baixa nos preços, porém com certa estabilização nos meses previstos.

\section{Referências}

BOX, G. E. P.; JENKINS, G. M. Time Series Analysis: Forecasting and Control. San Francisco,

Holden Day, 1976.

COSTA, C.K.F.; MAIA, S. F. Estratégia comercial para o agronegócio: estudo do impacto de barreiras sobre o setor produtivo brasileiro. Dissertação de mestrado em economia, Paraíba: UFPB, 150p. 2008.

GREENE, W. Econometric analysis. 5.ed. New Jersey: Prentice Hall, 2003. 
LIMA NETO, E. A.; LOUREIRO, J. Mineração de séries temporais e dados seqüenciais. Recife: UFPE, 2003.

LIMA, R.C.A; MIRANDA, S.H.G.; GALLI,F. FEBRE AFTOSA: Impacto sobre as exportações brasileiras de carnes e contexto mundial das barreiras sanitárias. Centro de Estudos Avançados em Economia Aplicada - ESALQ/ USP, 31p. 2005.

MIRANDA, S.H.G. Quantificação dos efeitos das Barreiras NãoTarifárias sobre as exportações brasileiras de carne bovina. Piracicaba. Tese de Doutorado - Escola Superior de Agricultura "Luiz de Queiroz”, Universidade de São Paulo, 237p. 2001.

MORETTIN, P.A.; TOLOI, C. M. Análise de Séries Temporais. $2^{\mathrm{a}}$ edição. São Paulo: Edgard Blucher, 2006.

PERRON, P. The Great Crash, the Oil Price Shock, and the Unit Roots Hypothesis. Econometrica, v. 57, n.6, p. 1361-1401, 1989.

ZIVOT, E.; ANDREWS, D. W. K. Further evidence on the great crash, the oil price shock and the unit-root hypothesis. Journal of Business and Economic Statistics, 10 (3), p. 251-270, 1992.

\footnotetext{
Abstract - The objective of this study was to identify some facts that had influenced the fatcow arroba prices received by producers as well as carrying through estimates of these prices for a period of four months beginning in May 2007. It was used methodology idealized by Box-Jenkins (1976). The results demonstrated that the series in question (01/1996-05/2007) presents a structural breaking in the referring period the January of 2004 , fact this that led to the cut of the same, being considered in the analysis the period February 2004 - May 2007, presenting 40 comments. Some events can have influenced the prices of the fatcow arroba in that moment and one that deserves prominence is the fact of that in period two infected areas of aphthous fever in the states of Amazonas and Pará had been detected what it caused the embargo of the exportations for Russia.

Keywords: Price, fatcow, aphthous fever.
} 
REVISTA DE ECONOMIA E AGRONEGÓCIO, VOL.6, $N^{\circ} 2$ 